\title{
ESTIMAÇÃO DAS FRAÇÕES DE DESVIO E ZONAS MORTAS A PARTIR DAS CURVAS DE DISTRIBUIÇÃO DE TEMPOS DE RESIDÊNCIA EM REATOR CONTÍNUO DO TIPO TANQUE AGITADO (CSTR)
}

\author{
B. R. BOTINHA ${ }^{1}$, H. V. de MIRANDA ${ }^{1}$ e F. T. VIEIRA ${ }^{1}$ \\ ${ }^{1}$ Universidade Federal do Espírito Santo, Campus de Alegre \\ E-mail para contato: hiago_vm@hotmail.com
}

\begin{abstract}
RESUMO - O presente trabalho apresenta a metodologia para a estimação da fração de desvio e zonas mortas a partir das curvas de distribuição de tempos de residência (DTR) em um reator CSTR, construídas a partir de dados experimentais, obtidos pela técnica estímulo-resposta de injeção do traçador. Utilizou-se um reator em escala de bancada e o fluxo contínuo de fluido foi mantido por bombas peristálticas. Avaliou-se a influência da velocidade de rotação e o efeito da presença de chicanas nas paredes internas do reator. A concentração de traçador foi determinada pela técnica colorimétrica, utilizando um espectrofotômetro. Obtiveram-se as curvas de distribuição de tempos de residência, de distribuição cumulativa e o tempo médio de residência. Com os dados experimentais, estimaram-se as frações de zonas mortas e caminhos preferenciais no interior do reator, utilizando o software Matlab. Observou-se um bom ajuste, com as curvas de DTR e distribuição cumulativa próximas das curvas teóricas para o reator ideal, podendo concluir que nas condições analisadas, o reator estudado atua próximo do comportamento ideal.
\end{abstract}

\section{INTRODUÇÃO}

O reator contínuo de tanque agitado (CSTR) é utilizado em reações que ocorrem em fase líquida quando é necessária a agitação intensa em processos de fluxo contínuo. O seu uso é muito difundido em escala industrial e laboratorial. Reatores CSTR são geralmente operados em estado estacionário e se considera que composição, temperatura e velocidade de reação são uniformes em todo seu interior. Dessa forma, a concentração de saída destes reatores é considerada como sendo igual à concentração dentro do tanque de reação (Fogler, 2009; Levenspiel, 2000).

Entretanto, nas condições reais de operação, o comportamento nos reatores CSTR se desvia do ideal, podendo resultar em contato não-efetivo e conversões mais baixas. Esses desvios podem ser causados pela formação de canais preferenciais ou pela criação de zonas mortas no tanque (Fogler, 2009; Levenspiel, 2000).

Um importante método de diagnosticar problemas relacionados ao perfil de escoamento e descrever o comportamento fluidodinâmico nos reatores reais é a partir da função distribuição de tempo de residência (DTR), que descreve o tempo que cada fração do reagente 
alimentado permanece dentro do reator. A DTR pode ser obtida, experimentalmente, a partir da injeção de um traçador (substância química inerte) no interior do reator e a subsequente medição da sua concentração na corrente de saída em função do tempo (Fogler, 2009).

O presente trabalho teve por objetivo operar um sistema composto por um reator CSTR com a injeção de traçador, para obtenção de curvas de distribuição de tempos de residência e curvas de distribuição cumulativa; comparar os comportamentos obtidos com simulações para o reator ideal com mistura perfeita; descrever o comportamento do reator a partir da avaliação da velocidade de rotação do impelidor e do efeito de chicana sobre o comportamento fluidodinâmico; e, a partir disso, estimar frações de desvio e zonas mortas para cada condição operacional.

\section{REVISÃO BIBLIOGRÁFICA}

Para descrever a DTR é utilizada a função $E(t)$, pela qual é possível caracterizar o tempo que diferentes frações de fluido permanecem no reator. Por meio da curva da função, é possível identificar problemas relacionados ao escoamento, tais como zonas mortas e caminhos preferenciais. Para uma perturbação de traçador do tipo pulso, que consiste na injeção repentina de todo o traçador na corrente de alimentação do reator, a função de distribuição de tempo de residência é descrita pela Equação 1 (Fogler, 2009; Levenspiel, 2000).

$$
E(t)=\frac{C(t)}{\int_{0}^{\infty} C(t) d t}
$$

A DTR normalizada, Equação 2, é obtida pela substituição da variável tempo pelo tempo adimensionalizado ( $\Theta)$. O termo $\Theta$ é calculado pela Equação 3.

$$
\begin{aligned}
& E(\Theta)=\tau E(t) \\
& \Theta=t / \tau
\end{aligned}
$$

Em que $\tau$ é o tempo de residência teórico do reator.

A função $F(t)$, Equação 4, descreve a fração de traçador na corrente de saída que permaneceu no reator por um período de tempo menor que $t$ (Fogler, 2009).

$$
F(t)=\int_{0}^{t} E(t) d t
$$

O tempo médio $\left(\mathrm{t}_{\mathrm{m}}\right)$, dado pela Equação 5 , é um importante parâmetro para caracterização do tempo de residência. Para um CSTR de mistura perfeita (ideal), o tempo médio da distribuição de tempo de residência é igual ao tempo de residência (Fogler, 2009). 


$$
t_{m}=\int_{0}^{\infty} t E(t) d t
$$

\section{METODOLOGIA}

\subsection{Procedimento Experimental}

A Figura 1 apresenta o aparato experimental utilizado durante os ensaios experimentais. Para proporcionar o fluxo contínuo no reator, foram utilizadas duas bombas peristálticas Logen Scientific modelo LS 2400 (1), as quais estavam acopladas ao reator de 3 litros (2) por meio de duas mangueiras de silicone (3) na entrada superior e na saída inferior do mesmo.

Figura 1 - Reator e bombas peristálticas utilizados nos ensaios experimentais.

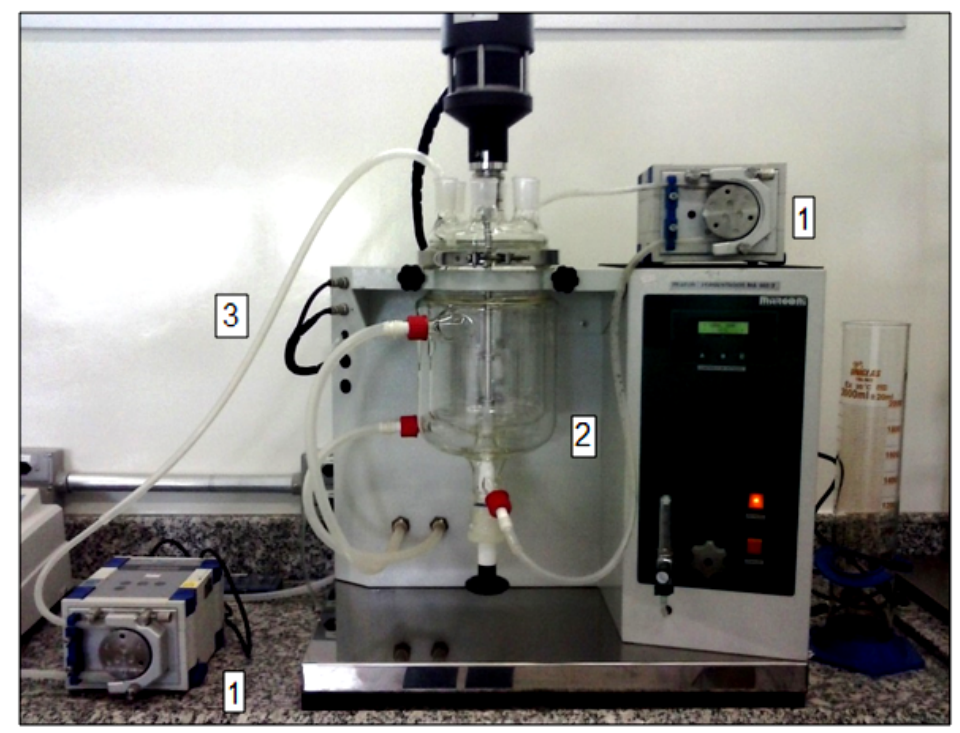

Os ensaios experimentais foram realizados com agitação contínua e vazão constante de $600 \mathrm{~mL} / \mathrm{min}$. A vazão foi mantida neste valor para que o tempo de realização dos experimentos não fosse muito longo. O reator/fermentador Marconi MA 502/3 estudado possui controle de agitação, também sendo possível a utilização de chicanas em suas paredes internas. Foram analisadas as influências da velocidade de rotação e da presença de chicanas sobre o comportamento da mistura dentro do reator.

No reator, a entrada do traçador permanganato de potássio foi do tipo pulso, sendo injetado rapidamente na entrada do mesmo, a fim de minimizar a dispersão deste, utilizando uma seringa preenchida com $5 \mathrm{~mL}$ da solução de traçador com concentração de $30 \mathrm{mg} / \mathrm{mL}$. As amostras do efluente com traçador foram coletadas com a utilização de tubos tipo Falcon de $15 \mathrm{~mL}$ na corrente de saída do reator em tempos pré-determinados, de $5 \mathrm{~s}$ a $26 \mathrm{~min}$. As concentrações dessas amostras foram obtidas por meio da medição de suas respectivas absorbâncias utilizando um espectrofotômetro Kazuaki modelo IL-227, previamente calibrado no intervalo de concentrações de 0 a $50 \mathrm{mg} / \mathrm{L}$. 
As velocidades de rotação do impelidor utilizadas foram de 200, 400, 500, 600 e $800 \mathrm{rpm}$. O erro experimental foi analisado no ponto central $(500 \mathrm{rpm})$, por meio da realização do experimento em triplicata, para avaliar o erro experimental. Embora não seja necessariamente verdade absoluta, esse nível de erro foi considerado o mesmo em toda faixa de rotação, a fim de reduzir o esforço experimental.

\subsection{Modelagem da Concentração de Traçador no Reator}

Para realizar a modelagem da concentração de traçador no efluente do reator foram considerados dois parâmetros: fração de zonas mortas $(\alpha)$ e de desvios $(\beta)$. Considerou-se o volume ocupado pelas zonas mortas como uma região separada do volume útil e que a vazão de desvio era uma corrente que não percorria o volume do reator. Os parâmetros $\alpha$ e $\beta$ foram, então, definidos pelas Equações 6 e 7, respectivamente.

$$
\alpha=\frac{\text { Volume de zonas mortas }}{\text { Volume do reator }}
$$

$$
\beta=\frac{\text { Vazão de desvio }}{\text { Vazão total }}
$$

A partir do balanço material para o traçador no reator CSTR e após manipulações algébricas, foi obtida a Equação 8. Esta foi utilizada para realizar as estimações de frações de zonas mortas $(\alpha)$ e desvios de fluido no reator $(\beta)$ com base nos dados experimentais, bem como realizar a simulação da concentração de traçador no reator em função do tempo. Para isto, foi utilizada a rotina de integração numérica ode 45 e a rotina de otimização fminsearch do software MATLAB.

$$
\frac{d C_{A}}{d t}=\frac{(1-\beta)^{2}}{\tau(1-\alpha)}\left(C_{A 0}-\frac{C_{A}-\beta C_{A 0}}{1-\beta}\right)
$$

Em que $\mathrm{C}_{\mathrm{A} 0}$ é a concentração de traçador na entrada do reator e $\mathrm{C}_{\mathrm{A}}$ é a concentração de traçador no efluente do reator.

\section{RESULTADOS E DISCUSSÃO}

Para exemplificação das curvas obtidas, na Figura 2 estão apresentadas as curvas de distribuição de tempos de residência normalizada $(E(\theta))$, distribuição cumulativa $(F(t))$ e dos valores de concentrações experimentais e simulados após a estimação dos parâmetros $\alpha$ e $\beta$, para a velocidade de rotação do impelidor de $500 \mathrm{rpm}$ na ausência de chicanas.

Em todas as condições operacionais avaliadas, observou-se que os pontos experimentais ficaram muito próximos do resultado teórico para o reator ideal, como é possível observar na Figura 2-(a) para a rotação de $500 \mathrm{rpm}$ no reator sem chicanas. As curvas teóricas de distribuição cumulativa também ficaram bastante próximas das curvas experimentais, como se observa na Figura 2-(b). Por meio desses resultados, foi possível afirmar que o reator CSTR é capaz de manter seu comportamento fluidodinâmico muito próximo do ideal em operações que envolvam fluidos com viscosidades similares a da água, em toda a faixa de velocidades testadas, com ou sem chicanas nas paredes de seu reservatório. 
Na Tabela 1, estão contidos os valores do tempo de residência teórico $(\tau)$, tempo médio de residência experimental $\left(t_{m}\right)$ e as frações de zonas mortas $(\alpha)$ e desvios $(\beta)$ estimadas para cada velocidade de rotação do impelidor para o reator com ausência de chicanas nas paredes internas.

Figura 2 - (a) DTR normalizada; (b) curva de distribuição cumulativa; (c) concentrações experimental e simulada de $\mathrm{KMnO} 4$ para rotação de $500 \mathrm{rpm}$ sem chicanas.
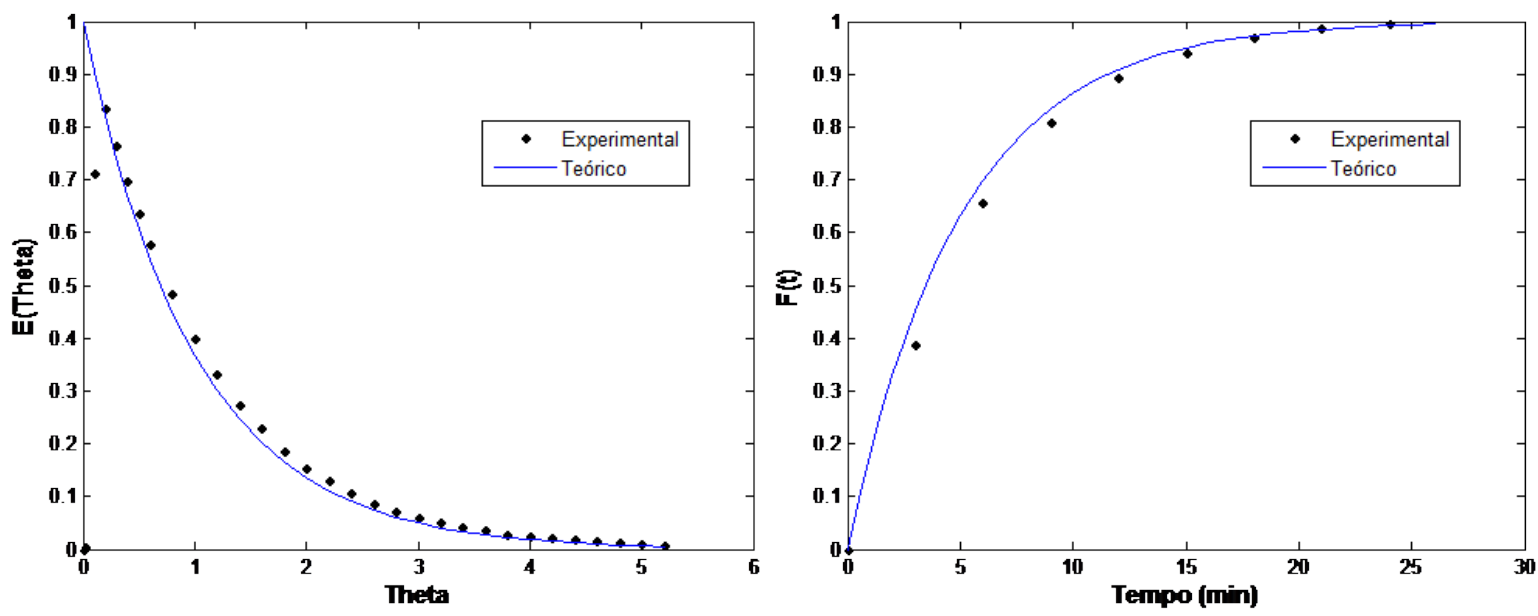

(a)

(b)

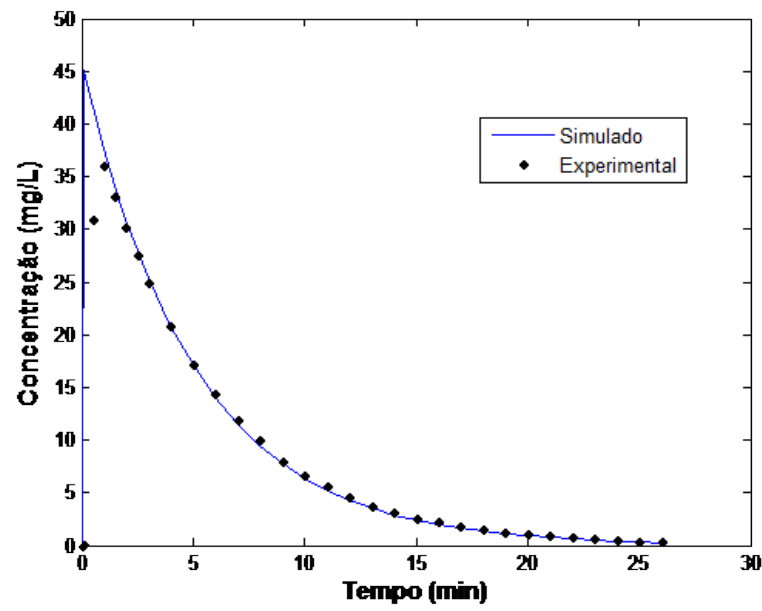

(c)

Tabela 1 - Resultados obtidos para o reator na ausência de chicanas.

\begin{tabular}{ccccc}
\hline Rotação $(\mathbf{r p m})$ & $\boldsymbol{\tau}(\mathbf{m i n})$ & $\boldsymbol{t}_{\boldsymbol{m}} \mathbf{( m i n )}$ & $\boldsymbol{\alpha} \mathbf{( \% )}$ & $\boldsymbol{\beta} \mathbf{( \% )}$ \\
\hline 200 & 5,00 & 5,73 & 0,94 & 10,58 \\
400 & 5,00 & 5,34 & 4,71 & 7,05 \\
500 & 5,00 & 5,52 & 5,33 & 6,71 \\
600 & 5,00 & 5,09 & 6,10 & 6,03 \\
800 & 5,00 & 5,18 & 5,95 & 6,13 \\
\hline
\end{tabular}


Os dados para o reator com presença de chicanas nas paredes internas estão apresentados na Tabela 2 .

Tabela 2 - Resultados obtidos para o reator com presença de chicanas.

\begin{tabular}{ccccc}
\hline Rotação $(\mathbf{r p m})$ & $\boldsymbol{\tau} \mathbf{( m i n )}$ & $\mathbf{t}_{\mathbf{m}} \mathbf{( \mathbf { m i n } )}$ & $\boldsymbol{\alpha} \mathbf{( \% )}$ & $\boldsymbol{\beta} \mathbf{( \% )}$ \\
\hline 200 & 5,00 & 5,49 & 5,00 & 6,92 \\
400 & 5,00 & 5,30 & 6,48 & 6,54 \\
500 & 5,00 & 5,64 & 3,84 & 7,75 \\
600 & 5,00 & 5,73 & 4,53 & 7,44 \\
800 & 5,00 & 5,75 & 3,16 & 8,63 \\
\hline
\end{tabular}

Para as curvas de concentração obtidas pelo modelo proposto e com os parâmetros estimados contidos nas Tabelas 1 e 2, observou-se que, para todas as condições operacionais, os valores estimados estão muito próximos dos pontos experimentais, de forma que se pode considerar que o modelo possui boa adequabilidade ao comportamento do reator e que a estimação dos parâmetros foi eficaz. Como se observa para uma das condições operacionais na Figura 2-(c), a curva de concentração de traçador obtida pela simulação do modelo foi capaz de prever os valores de quase todas as concentrações obtidas experimentalmente.

O tempo médio de residência experimental foi de $(5,52 \pm 0,06)$ min para a velocidade de $500 \mathrm{rpm}$ na ausência de chicanas, condição na qual os experimentos foram realizados em triplicata. Na hipótese dos desvios para as outras velocidades de rotação serem equivalentes a do ponto central $(500 \mathrm{rpm})$, é possível afirmar que o tempo médio nessa rotação possui valores distintos dos obtidos para as outras velocidades de rotação. O mesmo pode ser concluído para o processo a $500 \mathrm{rpm}$ na presença de chicanas, em que o tempo médio foi $(5,64 \pm 0,04) \mathrm{min}$.

Foram realizados o teste $\mathrm{F}$ e o teste $\mathrm{t}$ de Student para avaliar se havia equivalência entre os valores de tempo médio de residência para os experimentos na ausência e na presença de chicanas, na rotação de $500 \mathrm{rpm}$. Pelos resultados dos testes, foi possível concluir que não houve diferença estatística entre os valores obtidos. Logo, a influência das chicanas nas paredes do reator para experimentos com água, ou fluidos com viscosidades semelhantes, a 500 rpm, não é significativa.

\section{CONCLUSÃO}

O reator CSTR estudado apresentou, em todas as condições operacionais avaliadas, comportamento fluidodinâmico muito próximo do esperado para o reator ideal, com pouca ocorrência de desvios e zonas mortas. Pode-se concluir que a estimação dos parâmetros frações de zonas mortas e desvios foi eficaz e que o modelo obtido foi capaz de prever satisfatoriamente os valores das concentrações experimentais nas condições analisadas.

\section{REFERÊNCIAS}


FOGLER, H. S. Elementos de engenharia das reações químicas. 4. ed. Rio de Janeiro: LTC, 2009.

LEVENSPIEL, O. Engenharia das reações químicas. 3. ed. São Paulo: Editora Edgard Blucher, 2000. 